http://jmscr.igmpublication.org/home/

ISSN (e)-2347-176x ISSN (p) 2455-0450

crossref DOI: https://dx.doi.org/10.18535/jmscr/v8i6.95

Journal Of Medical Science And Clinical Research

\title{
Actinomycosis affecting the maxilla: A case report of rare occurrence
}

\author{
Authors \\ Dr Amrita Samanta ${ }^{1 *}$, Dr Nandan Rudra Paul ${ }^{2}$ \\ ${ }^{1}$ Department of Oral and Maxillofacial Pathology, The Oxford Dental College, Bangalore, India \\ ${ }^{2}$ Department of Oral and Maxillofacial Surgery, The Oxford Dental College, Bangalore, India \\ *Corresponding Author \\ Dr Amrita Samanta \\ Assistant Professor, Department of Oral \& Maxillofacial Pathology, \\ The Oxford Dental College, $10^{\text {th }}$ Milestone, Hosur Road, Bommanahalli, 560068, Bangalore, India
}

\begin{abstract}
Background: Actinomycosis is a rare, painless, indolent, granulomatous infection caused by Actinomyces spp. The varied clinical presentation often can be misdiagnosed which can later lead to complications. Involvement of the oral cavity can mimic a gingival or periodontal abscess. This article presents a clinical case of actinomycosis in the posterior maxilla with not-so-pathognomic clinical presentation but a complete successful resolution of the infection.

Methods: A 36-year old male patient presented with mobile tooth and difficulty in mastication, without pain. Intraoral examination and periapical radiograph was done for the affected tooth no. 26. Surgical extraction and debridement was carried out under local anaesthesia. Bone graft was placed and the surgical site was covered with buccal advancement flap. Patient was put on Amoxycillin (875 mg) + clavulanic acid $(125 \mathrm{mg})$ twice a day for a period of 5 days. After the histopathological examination confirmed the diagnosis of Actinomycosis, the antibiotic regime was prolonged for another five weeks. Case reports on actinomycosis were searched on Google Scholar and other databases. Summarisation of data and evaluation was carried out according to a critical appraisal checklist for case reports.

Results: Satisfactory post operative healing of the surgical site was noted after six weeks of follow up. Intra oral peri-apical radiograph revealed increased bone height below the floor of maxillary sinus after antibiotic therapy for a period of 6 weeks.

Conclusion: The surgical curettage of the extraction socket along with long term penicillin therapy is quintessential for the best outcome.

Keywords: actinomycosis, antibiotic, giant cells, graft, granuloma, maxilla.
\end{abstract}

\section{Introduction}

Actinomycosis is chronic, rare granulomatous disease, caused by anaerobic or microaerophilic Gram positive filamentous bacilli, from the Actinomycetaceae family (genus Actinomyces) ${ }^{[1]}$. More than 30 species has been isolated, among which $A$ israelii has been detected as the most common human commensal as well as pathogen $^{[2]}$. They comprise the oral microbiota within gingival crevices, periodontal pockets, dental plaque, carious teeth, tonsillar crypts ${ }^{[3]}$. Studies have suggested that actinomyces establish 
an infection by inhibiting host defence mechanism, although the exact pathway still remains unknown ${ }^{[3]}$. The infection grows continually through anatomic barriers damaging through mucosal tissue, leading to abscesses and fistulous tract. ${ }^{1}$ Moreover, the pathogenicity is enhanced by the "companion microbes" which also invade the affected tissues ${ }^{[4]}$. Immunocompromised patients are found to be at higher risk, though the role of immunosupression is unclear ${ }^{[1,2]}$. In the oral cavity, actinomycosis can mimic an abscess of periodontal origin, gingival abscess or chronic periodontitis. Thus, a prudent diagnosis is required as the treatment is quite demanding. The tradition of treatment revolves around penicillin in high doses and prolonged periods after adjunct surgical intervention. This mandates to tailor the treatment based on the involved anatomical site, extension of the infection, and response to the antibiotics.

\section{Case Description and Results}

A male patient aged 36 years reported at our clinic OPD with the chief complaint of a 'shaking' tooth in the left upper back region of mouth. The patient gives the history of the mobile tooth, which is the left upper $1^{\text {st }}$ molar, to be mobile since 3 years and with a gradual increase in mobility. This was not associated with any sort of pain. However, there was difficulty in chewing. Extraoral examination of face and the head and neck region revealed no remarkable abnormality. On intraoral examination, it was found that tooth no. 26 was having grade III with furcation involvement on the buccal aspect along with distobuccal root exposure.The tooth was depressible in the socket. There was no pus discharge or tender on percussion present. The intraoral periapical radiograph (Figure 1) reveals circum-apical well defined radiolucency around 26, extending from the cement-enamel junction of the tooth till $2 \mathrm{~mm}$ below the floor of the maxillary sinus. This depicts extensive bone loss around tooth no. 26.

Treatment was done following complete aseptic protocol. Under local anaesthesia, extraction of 26 was carried out along with complete debridgement and curettage of the involved socket, only to remove a thick band of soft tissue. The surgical site was packed with allogenous bone graft ( $\beta$ tricalcium phosphate + hydoxyapatite) crystals and covered with buccal advancement flap. The soft tissue along with the tooth was sent for biopsy. Histopathological examination reveals parakeratinised hyperplastic epithelium with focal areas of spongiosis. Many moderately sized eosinophilic abscesses (Figure 2) containing hematoxyphilic filaments of bacteria with eosin stained peripheral club-shaped ends are seen depicting the 'sun ray' appearance. The connective tissue shows increased inflammatory cell infiltrate (Figure 3) comprising of neutrophils and lymphocytes. Few giant cells are seen. Fragments of bone are evident. This confirms the diagnosis of Actinomycosis. Patient was recalled at an interval of 1 day followed by weekly follow up for 6 weeks. Patient was under oral medication of Amoxycillin (875 mg) plus clavulanic acid $(125 \mathrm{mg})$ twice a day for a period of 6 weeks, Metronidazole $(400 \mathrm{mg}$ ) thrice a day for 5 days along with analgesics for 5 days, which was further prolonged for another continuous five weeks. Patient was instructed to use betadiene mouthwash from the second day of surgery. Post 6 week follow up period, patient was assessed and it was found that the surgical site (Figure 4) has healed up completely. On radiograph (Figure 5), good bone height can be appreciated.

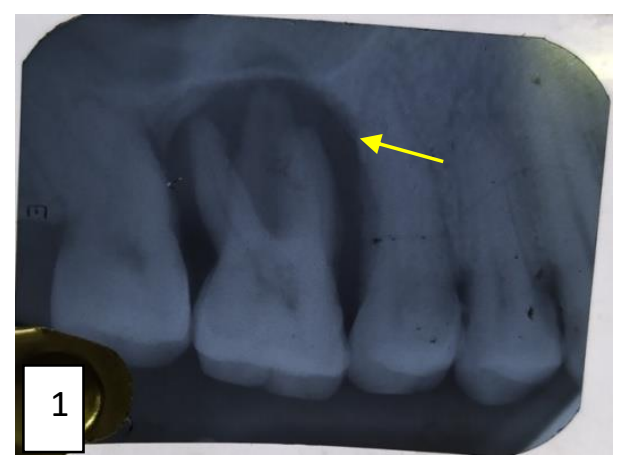

Figure 1: Pre-operative Intraoral periapical radiograph showing a circumferential radiolucency involving the pericemental area of tooth no. 26 involving the floor of maxillary sinus. 


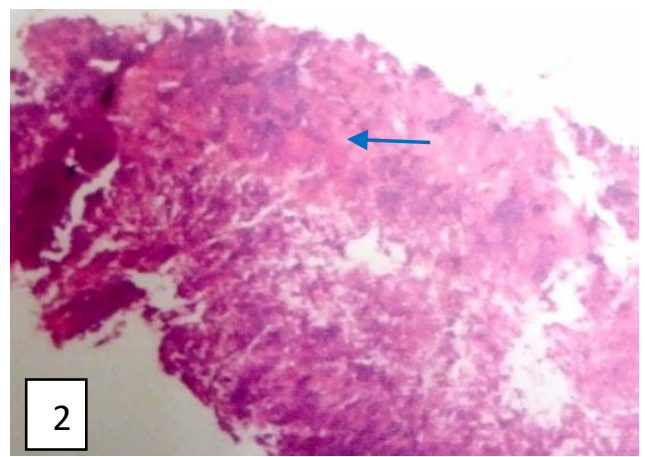

Figure 2: Histopathology showing eosinophilic round to oval areas of abscess formation (blue arrow) and bacterial colonies (hematoxylin and eosin stain, original magnification $\mathrm{x} 40$ ).

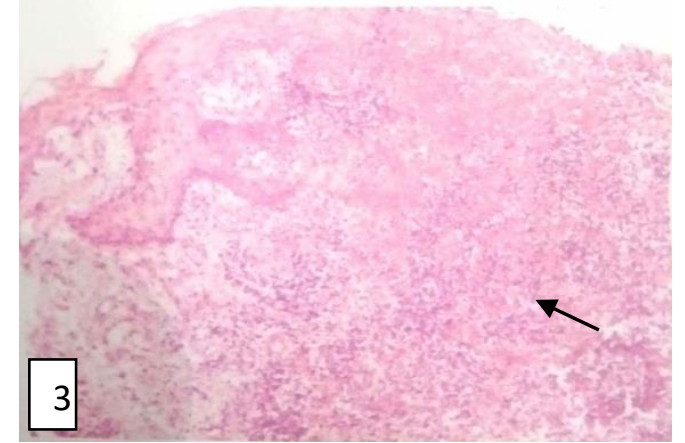

Figure 3: Histopathology showing a sea of inflammatory cell infiltrate (black arrow) in the connective tissue (hematoxylin and eosin stain, original magnification $\mathrm{x} 40$ ).

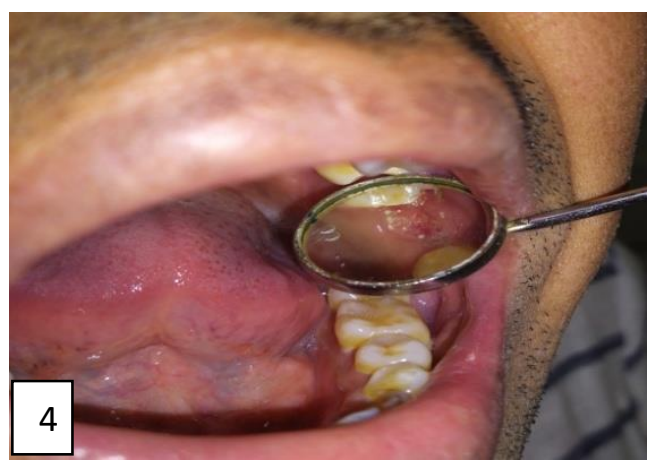

Figure 4: Satisfactory healing of the surgical site after 6 weeks follow up.

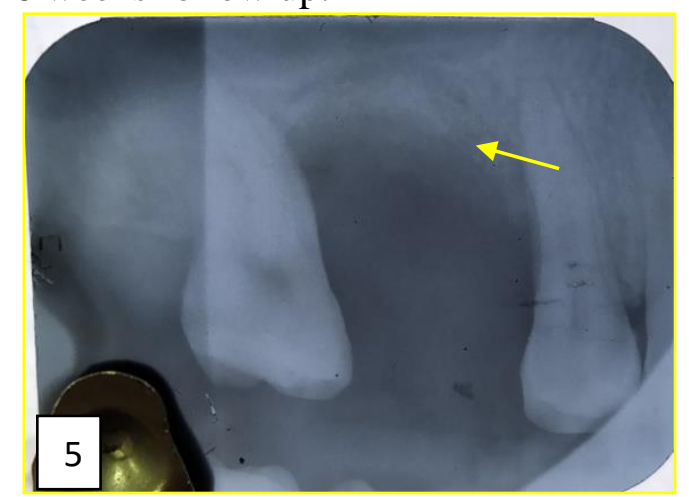

Figure 5: Post-operative Intraoral periapical radiograph showing perfect healing of the site with adequate bone height (yellow arrow).

\section{Discussion}

Actinomyces were initially believed to be a fungi due to the presence of filamentous structures and was included in mycology which after serological and biochemical study revealed broken microfilaments of bacillary and coccoid forms and confirmed to be a bacteria ${ }^{[5,6,8]}$. It is a commensal in various parts of the human body including oral cavity, thorax, abdomen and vagina ${ }^{[1,7]}$. Our case has been reported in the maxilla pertaining to tooth no. 26. Actinomyces, being a saprophyte in the oral microbiota causes a painless, granulomatous inflammatory disease that may be suppurative, exhibiting local aggressiveness and destroying the tissue components ${ }^{[5]}$. In our case, the patient reported with a painless, mobile (grade I) tooth since 3 months which progressively became more mobile (grade III) over a period of time leading to difficulty in mastication. This reveals local destruction of supporting structures of tooth in such short duration of time depicting the aggressiveness of the disease. There was no pus discharge or tenderness to percussion. The commonest species which have been isolated from actinomycosis infections are A. israelii, $A$. naeslundii, $A$. viscosus and A. Meyeri ${ }^{[6,7]}$. Classification of Actinomycosis clinically is based upon the infected anatomical location: oral, cervicofacial, thoracic, abdominopelvic, central nervous system, musculoskeletal, and disseminated $^{[2]}$. Middle aged males are most commonly affected ${ }^{[6]}$. This case is also regarding a male patient aged 36 years. Predisposing factors for the occurrence of oral actinomycosis can be attributed to carious tooth, gingivitis, oral mucosal trauma from tooth extraction, local tissue damage by neoplasm or radiation, surgical procedures. Related factors include malnutrition, diabetes, immunocompromised status and chronic alcoholism $^{[4]}$.

Actinomycosis produces a localised inflammatory reaction which is a granuloma with central suppuration having peripherally giant cells and fibroblasts. The abscess in the centre contains yellow sulphur granules with radiating filaments 
(Splendore-Hoeppli phenomenon) mimicking ray fungus, a misnomer ${ }^{[8]}$. In our case, the tissue retrieved from the extraction socket was fibrous in consistency. Typical granulomatous reaction with central abscesses surrounded by numerous leucocytes and many giant cells were seen. The round to oval colonies were made up of haematoxyphilic filaments with peripheral eosinophilic club-shaped ends, which is characteristic of actinomycosis. Our case could not effectively demonstrate the presence of sulphur granules. The history of infection and the clinical presentation of the patient did not allow us to seek a laboratory culture.

Literature suggests, beta-lactam antibiotics especially penicillin $\mathrm{G}$ or amoxicillin are highly effective and are the drug of choice for treating actinomycosis. Piperacillin-tazobactam, imipenem, and meropenem are also effective and active against Actinomyces spp. However, limited application of such broad-spectrum antibiotics is advised to avoid future drug resistance ${ }^{[4]}$. In our case, the wound healing responded satisfactorily to the oral amoxicillin (875mg)+ clavulanic acid $(125 \mathrm{mg}) \mathrm{BD}$, combined with metronidazole (400mg) TID, with the allogenous bone graft insitu leading to increase in bone height radiographically after a span of 6 weeks.

\section{Conclusion}

Actinomycosis is truly under-reported due to diagnostic difficulties, missing out on proper investigative modalities, and the empirical use of antibiotics. ${ }^{1}$ Clinician awareness of the disease plays a pivotal role in prevention of the disease by early diagnosis, thereby limiting the more complicated forms like pulmonary or cental nervous system involvement. Improvement of oral hygiene usually is the key to stay away from complicated infections.

\section{Acknowledgments and Disclosure Statements}

The authors report no conflicts of interest related to this study.

\section{References}

1. Bonnefond S, Catroux M, Melenotte C, Karkowski L, Rolland L, Trouillier S, Raffray L. Clinical features of actinomycosis: A retrospective, multicenter study of 28 cases of miscellaneous presentations. Medicine 2016; 95:24. [http://dx.doi.org/10.1097/MD.000000000 0003923].

2. Wong VK, Turmezei TD, Weston VC. Actinomycosis. BMJ 2011;343:d6099. [ doi: 10.1136/bmj.d6099].

3. Moturi K, Kaila V. Cervicofacial actinomycosis and its management. Ann Maxillofac Surg 2018;8:361-4.[ DOI:10.4103/ams.ams_176_18].

4. Valour F, Sénéchal A, Dupieux C, Karsenty J, Lustig S, Breton P, Gleizal A, Boussel L, Laurent F, Braun E, Chidiac C, Ader F, Ferry T. Actinomycosis: etiology, clinical features, diagnosis, treatment, and management. Infection and Drug Resistance 2014:7 183-197. [http://dx.doi.org/10.2147/IDR.S39601].

5. Thukral R, Shrivastav K, Mathur V, Barodiya A, Shrivastav S. Actinomyces: a deceptive infection of oral cavity. J Korean Assoc Oral Maxillofac Surg 2017;43:282285.

[https://doi.org/10.5125/jkaoms.2017.43.4. 282].

6. Sivapathasundaram B. Bacterial infections of the oral cavity. Shafer's Textbook of Oral Pathology. 8th ed. Elsevier 2017; pp. 302-303.

7. Khodavaisy S, Zibafar E, Hashemi SJ, Narenji H, Daie Ghazvini R. Actinomycosis in Iran: Short Narrative Review Article. Iranian J Publ Health, Vol. 43, No.5, May 2014, pp. 556-560.

8. Mohan H. Granulomatous Inflammation. Essential Pathology for Dental Students. $2^{\text {nd }}$ ed. Jaypee 2002; pp. 123-124. 